\title{
Blending liquid into a flowing dry granular material
}

\author{
Guillaume Saingier $^{1, \star}$, Alban Sauret ${ }^{1}$, and Pierre Jop ${ }^{1}$ \\ ${ }^{1}$ Surface du Verre et Interfaces, UMR 125 CNRS/Saint-Gobain, 93303 Aubervilliers, France
}

\begin{abstract}
We study experimentally the growth dynamics of a horizontal wet granular aggregate produced by accretion when a dry granular jet impacts a wet substrate. The tomographic imaging demonstrates that the wet aggregate is fully saturated and its cohesion is related to the capillary suction due to the pressure drop at the liquid/air interface. We highlight that the accretion process is characterized by two different growth dynamics depending on the hydrostatic depression in the material. At low depression, the growth dynamics exibits a "diffusive" regime whereas the dynamics becomes linear for higher depressions. A competition between the viscous displacement of the fluid into the granular material and the sticking dynamics is proposed to understand the transition in the growth velocity.
\end{abstract}

\section{Introduction}

Granular materials are commonly used as raw materials in the building industry [1]. In particular, the preparation of many building materials such as concrete, mortar or plaster requires mixing granular matter with liquid and additives in order to obtain new chemical or physical properties. For a large added volume of water, a mixing stage easily produces a homogeneous granular suspension in which the grains freely move in the interstitial fluid. By contrast, the addition of smaller amounts of liquid provides a strong cohesion in the material through the formation of liquid bridges between grains [2-4]. For intermediate liquid amounts, spatial heterogeneities can appear during the blending process and generate bubbles or saturated aggregates, which will locally impact the mechanical properties of the final material. Most studies on wet granular matter are focused on the fluid flow dynamics and the repartition of the liquid into a static granular packing $[5,6]$. In this case, the material can be assimilated to a porous medium. Therefore, the liquid transport is essentially led by capillarity in the porosity of the material and exhibits well-known imbibition dynamics described in 1D by the Lucas-Washburn law [7].

Recently, dynamical situations were studied through experiments of granular towers built by dripping a granular suspension on a dry substrate [8] or by pouring dry sand on a wet substrate [9]. A cohesive granular structure is erected in each situation but the physcial origin and the growth dynamics is different. On one side, the tower growth is related to a competition between liquid drainage and dripping frequency. On the other, the dynamics is limited by the accretion of dry grains on the wet structure. However, this accretion process is still not understood and the origin of the growth dynamics and the capture mech-

\footnotetext{
^e-mail: guillaume.saingier@saint-gobain.com
}

anism were not explained. Here, we investigate the accretion process between liquid and grains, using a model experiment of liquid incorporation into a flowing granular material. We focus on an experiment of accretion at a constant height to remove the effect of gravity and keep the hydrostatic pressure constant in the material. We present an experimental set-up that allows modifying the hydrostatic pressure in the wet granular material and highlighting the growth regimes.

\section{Experimental set-up}

We performed experiments of granular accretion using spherical glass beads of diameters $d_{g}=300-350 \mu \mathrm{m}$ poured at constant flow rate and constant velocity over a wet substrate composed of the same beads (see Fig. 1). The wet granular layer of height $h_{g}=10 \mathrm{~mm}$ is prepared in a polypropylene tube of $10 \mathrm{~mm}$ diameter connected to a water tank to soak the grains. The wet substrate is separated from the liquid by a porous membrane and the water level can be tuned to modify the hydrostatic pressure into the granular substrate. We note $\Delta h$ the distance between the water level and the top of the wet substrate. Dry beads are poured using an inox funnel at a constant flow rate $\left(Q_{g}\right.$ $=1.1 \mathrm{~g} / \mathrm{s})$. The funnel is connected to a inox tube of length $L=200 \mathrm{~mm}$ with a flexible polyethylene end to inject the grains with an angle of about $45^{\circ}$. The granular aggregate grows horizontally on a thin plexiglas plate of width $w=$ $3 \mathrm{~mm}$ to hold it while avoiding the accumulation of dry grains in the front of the aggregate during the experiments. The extremity of the tube is held at $10 \mathrm{~mm}$ above the plexiglas plate such that the grains kept a constant velocity $\left(v_{g}\right.$ $\simeq 1.6 \mathrm{~m} / \mathrm{s}$ ) to impact the tip of the aggregate. The grain container is placed on a translating support allowing us to shift it while the aggregate is growing along the support such that the distance between the growing aggregate and 


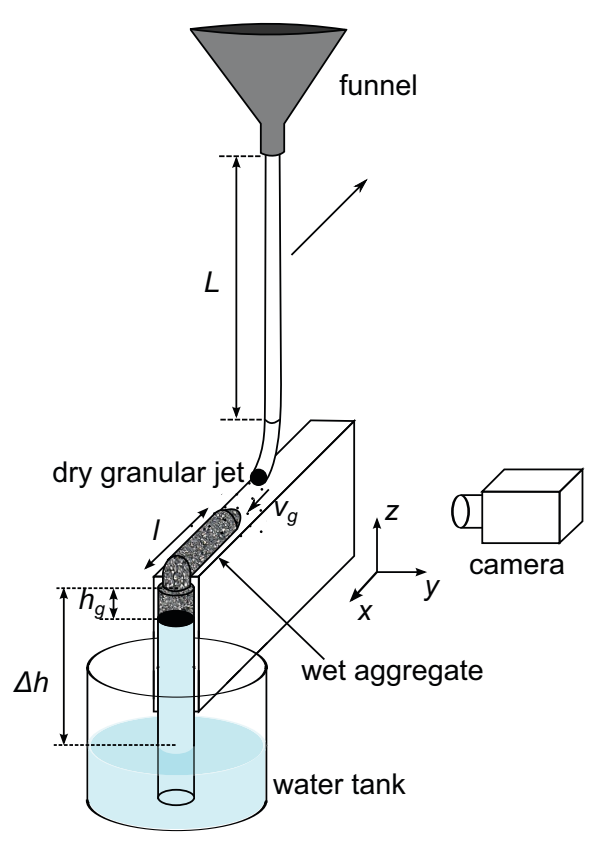

Figure 1. Schematic of the experimental set-up.

the extremity of the tube remains constant. The growth of aggregate is filmed at low acquisition frequency $\left(f_{\text {acq }}=\right.$ $0.5 \mathrm{~Hz}$ ) with a CCD camera and analyzed by image processing with ImageJ and Python scripts $[10,11]$. By using this experimental set-up, homogeneous aggregates are built horizontally.

\section{Experimental results}

In a typical experiment, the granular jet is oriented toward the wet substrate and an aggregate grows horizontally on the support. The width of structure is about the width of the support and the section remains nearly constant in each experiment. By increasing the height $\Delta h$, the growth dynamics can be changed drastically. To understand this phenomenon, we proposed to study the internal structure of the wet aggregate and its growth dynamics.

\subsection{Microstructure of the aggregate}

To investigate the microstructure of the aggregate, X-ray tomography experiments were carried out with a laboratory tomograph available at Saint-Gobain Research. The $\mathrm{X}$-ray radiations were generated by an electron gun bombing a tungsten plate which produces photons at a peak energy of $40 \mathrm{kev}$. The spatial resolution was $7.5 \mu \mathrm{m}$ and the detector fields of view was $13 \mathrm{~mm}$ x $10 \mathrm{~mm}$. To increase the contrast between the different phases (grains, liquid and air), an aqueous zinc iodide solution, of $0.5 \mathrm{~g} / \mathrm{mL}$, was used as liquid to build the aggregate [6]. A tomographic acquisition consisted of 1395 projections acquired in about $40 \mathrm{~min}$ and equally separated to cover a total angle of $180^{\circ}$.

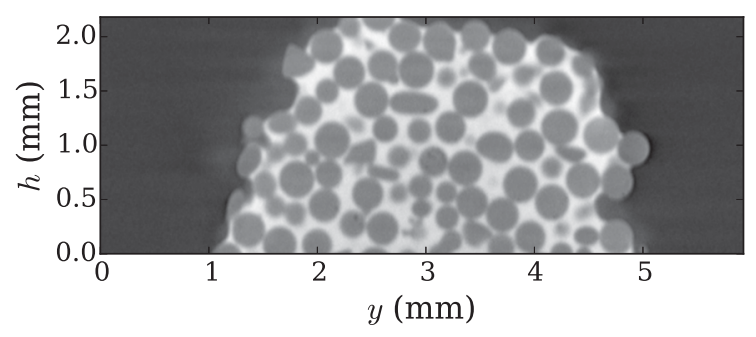

Figure 2. Cross-section through a 3D tomogram of a wet aggregate. The liquid is doped by adding zinc iodide to improve the contrast between the different phases. The liquid is colored in white whereas the glass beads appear in gray and the air in black.

The 3D reconstruction was performed from the set of $2 \mathrm{D}$ projection using the filtered backprojection algorithm.

The analysis of the tomographic reconstructions shows that the wet aggregate is fully saturated without almost no bubble in the granular assembly (see Fig. 2). The aggregate is in the capillary state and the cohesive interactions between the particles result from the capillary depression at the interface with the air. From this observation, we conclude that the hydrostatic pressure is the same at each point of the horinzontal aggregate growing at constant $\Delta h$. At the liquid/air interface, the capillary drop associated to the local curvature of the menisci balances the hydrostatic pressure:

$$
p_{0}-\rho g \Delta h=p_{0}-\frac{2 \gamma \cos \theta}{r_{c}},
$$

where $\gamma=72 \mathrm{mN} / \mathrm{m}$ is the surface tension of the water, $\theta$ is the contact angle of water on a glass bead and $r_{c}$ is the radius of curvature of the meniscus at the interface. Consequently, the local curvature of a meniscus increases with $\Delta h$ in our experiment.

\subsection{Growth dynamics}

We observed that the aggregate grows slower when $\Delta h$ increases, so that the accretion process is less efficient in this case. An example of snapshots from the experiments is presented in Fig 3. By performing several tests at different altitude and by measuring the length $\ell$ of the wet granular structure in function of the time $t$, we can determine the growth dynamics of the aggregate. These curves are plotted in Fig. 4 for different $\Delta h$ at $Q_{g}$ and $v_{g}$ constant. A change of growth dynamics is clearly observed when $\Delta h$ increases with a transition from a "diffusive" regime where $\ell$ is proportional to $t^{1 / 2}$ to a linear regime where $\ell$ is proportional to $t$.

To understand the existence and the difference between the two regimes, we consider the local mechanism of the aggregate growth. The aggregate is built by trapping dry grains at the liquid interface of the wet material.The liquid needs to penetrate into the granular packing until being accessible to the granular jet. When $\Delta h$ is small, the radius of curvature of the menisci is large and we can assume that 


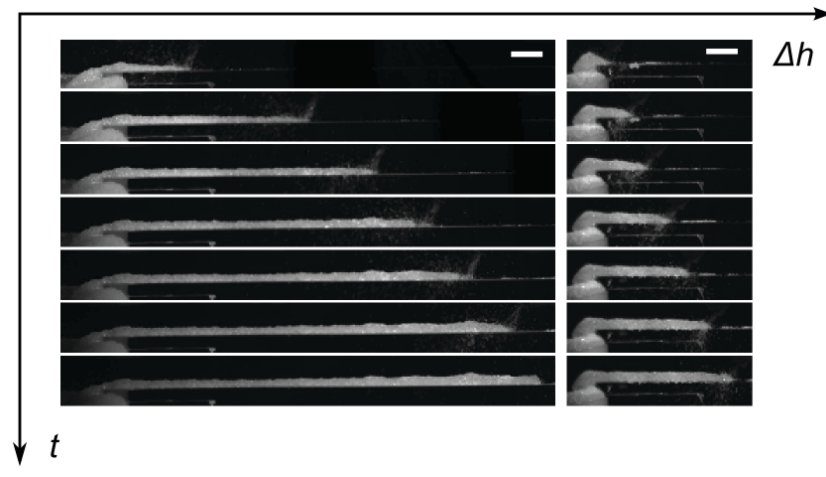

Figure 3. Snapshots of the growing process at different times and for two altitudes $\Delta h$. (left) $\Delta h=0.5 \mathrm{~cm}$ - (right) $\Delta h=4.9$ $\mathrm{cm}$. Snapshots are seperated by $40 \mathrm{~s}$. Scale bar is $1 \mathrm{~cm}$.

the liquid is available to catch a large fraction of grains. The growth rate of the aggregate is limited by the viscous displacement of the fluid into the granular packing until it reaches its equilibrium position. We can model this liquidlimited regime with the Darcy's law without gravity. The Lucas-Washburn law is obtained:

$$
\ell_{v i s c}(t)=\sqrt{\frac{2 k \Delta p t}{\eta}},
$$

where $k$ is the permeability of the packing (in $\mathrm{m}^{-2}$ ), $\eta$ is the dynamic viscosity (in Pa.s) and $\Delta p=p_{c}-\rho g \Delta h$ is the capillary pressure reduced by the hydrostatic depression. The pressure $p_{c}$ is defined as $2 \gamma \cos \theta /\left(\rho_{l} r_{p}\right)$ where $r_{p}$ is the pore radius. This "diffusive law" is qualitatively in agreement with the first regime identified in our results (see Fig. 4). For large $\Delta h$, the radius of curvature of the menisci is reduced and the liquid is less accessible to the grains impacting the aggregate. In this case, the efficiency of the capture process limits the growth of the aggregate. Considering the growth rate as the ratio of grains stuck over the total amount of grains impacting the aggregate,

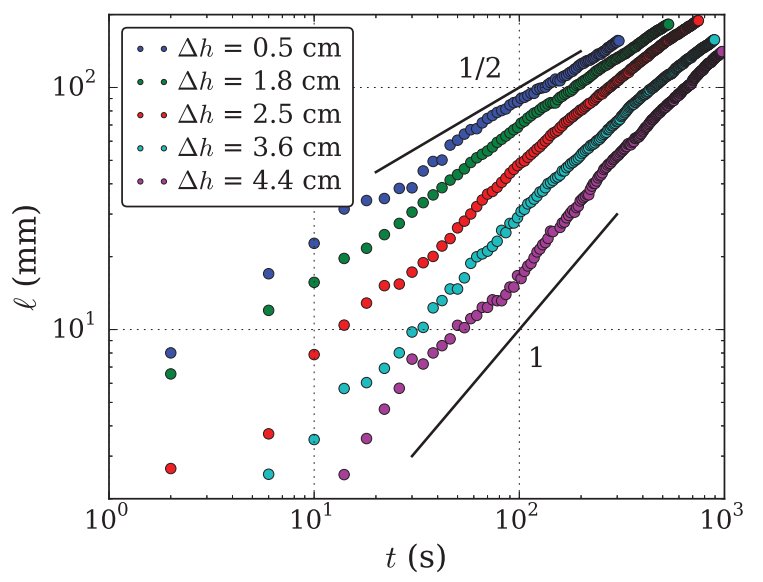

Figure 4. Time evolution of the length of the aggregate for several $\Delta h$ in log-log representation. The power laws lines curves are indicative.

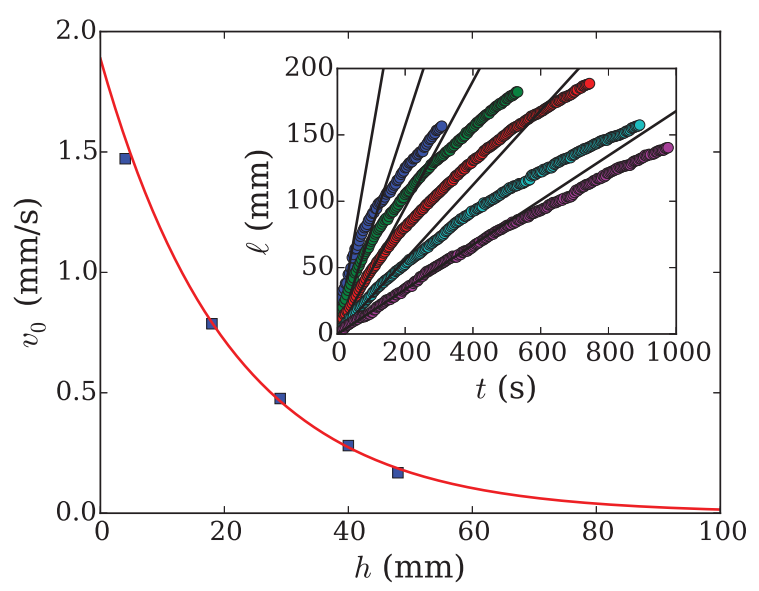

Figure 5. Evolution of the initial velocity of growth as a function of the distance $\Delta h$ between the water level and the top of the aggregate. The solid line is obtained by fitting the experimental data with an exponential expression. Inset: Length of the aggregate $\ell$ as a function of the time $t$ for different $\Delta h$.

we can write:

$$
\frac{\mathrm{d} \ell}{\mathrm{d} t}=\frac{Q_{g}}{\rho_{s} \phi S} P_{c a p t},
$$

where $\phi$ is the compacity of the packing, $S$ is the section of the aggregate and $P_{\text {capt }}$ is the ratio of stuck grains over the total injected grains. Assuming that $P_{\text {capt }}$ is constant at fixed $\Delta h$, the length of the aggregate grows in the capturelimited regime as:

$$
\ell_{\text {capt }}(t)=\frac{Q_{g}}{\rho_{s} \phi S} P_{\text {capt }} t .
$$

In summery, the "diffusive" behavior corresponding the liquid-limited regime and the linear behavior corresponding to the capture-limited regime are qualitatively plotted on the Fig. 4.

To estimate the efficiency of the accretion process at different altitude $\Delta h$, we derived the initial growth velocity $v_{0}$ of the aggregate. Indeed, the beginning of the aggregate growth is limited by the capture process on the wet substrate since the liquid is already available. The evolution of $v_{0}$ is plotted in Fig. 5 as a function of $\Delta h$. We observe that $v_{0}$ decreases with the altitude and is well-fitted by an exponential expression:

$$
v_{0}(h)=v^{\star} \exp \left(-\frac{\Delta h}{h^{\star}}\right),
$$

where $v^{\star}$ is the capture-limited growth velocity at $\Delta h=0$ and $h^{\star}$ is the length scale of variation of the sticking probability. Similar variations were observed for the growth velocity of a vertical granular towers built upon the accretion from a dry diluted granular jet [9]. The value of $h^{\star}$, computed from pressure-controlled experiments of horizontal aggregate, is compatible with measurements performed on vertical granular towers. This result indicates that the logarithmic law observed in the growth of vertical aggregate is linked to the variation of the hydrostatic pressure in the wet material. 


\section{Discussion}

The transition between the two growth regimes is expected when the growth rates associated to each limiting mechanism are comparable. For a constant $\Delta h$, at the beginning of the growth, the capture of dry grains is slow enough, compared to the liquid transfer, and limits the growth rate. As the length of the aggregate increases, the viscous transfer of liquid slows down and becomes limiting for the growth rate. Equating the capture and viscous growth rates leads to a critical length $L_{c}$ determining the linear-diffusive transition and defined as:

$$
L_{c}=\frac{k}{2 \eta} \frac{\rho_{s} \phi S}{Q_{g} P_{c a p t}} \Delta p .
$$

This transition drastically depends on the efficiency of the accretion process, which appears to decrease with the hydrostatic depression, controlled by $\Delta h$. Consequently, the transition length $L_{c}$ increases when $\Delta h$ increases because of the decrease of the sticking probability. For transitional values $(\Delta h=1.8 \mathrm{~cm}, \Delta h=2.5 \mathrm{~cm})$, an intermediate exponent between $1 / 2$ and 1 is identified, corresponding to the smooth linear-diffusive transition. Furthermore, for low values of $\Delta h(\Delta h<1 \mathrm{~cm})$, the critical length is close to the height $h_{g}$ of the substrate and the transition is not observed. In this case, nearly all the growth dynamics is dominated by the viscous displacement of liquid. Further investigations are now required to identify the impact of the fluid properties (surface tension, viscosity) and the grains velocity on the sticking probability.

\section{Conclusion}

The blending of liquid into a dry granular flow is considered in a model situation to create a wet aggregate growing by granular accretion at the interface between dry and wet grains. The tomographic imaging confirmed that the aggregate is in the capillary regime and its cohesion is related to the capillary suction associated to the pressure drop at the liquid/air interfaces. Our experimental set-up allowed us to control the hydrostatic pressure in the wet substrate to isolate the accretion process. We highlighted two regimes of accretion are identified associated to the fluid flow into the porous matrix and the efficiency of the grains capture process. In particular, the sticking probability is found to be constant at a fixed height. It confirms the importance of the hydrostatic depression in the dynamics and the locality of the capture mechanism. Our work may be useful for industrial applications to improve or control agglomeration processes such as the wet granulation.

\section{Acknowledgements}

We are grateful to William Woelffel for his help and his advice for the tomographic acquisitions.

\section{References}

[1] J. Duran, Sands, Powders, and Grains: An Introduction to the Physics of Granular Materials (SpringerVerlag, New-York, 2012).

[2] S. Herminghaus, Adv. Phys. 54, 221-244 (2005).

[3] N. Mitarai \& F. Nori, Adv. Phys. 55, 1-45 (2006).

[4] A. Kudrolli, Nat. Mater. 7, 175-175 (2008).

[5] M. Reyssat, L. Y. Sangue, E. A. Van Nierop \& H. A. Stone, Europhys. Lett. 86, 56002 (2009).

[6] M. Scheel, R. Seeman, M. Brinkman, M. di Michel, A. Sheppard, B. Breidenbach \& S. Herminghaus, Nat. Mater. 7, 189 (2008).

[7] W. Washburn, Phys. Rev. 17, 273 (1921).

[8] J. Chopin \& A. Kudrolli, Phys. Rev. Lett. 107, 208304 (2011).

[9] F. Pacheco-Vázquez, F. Moreau, N. Vandewalle \& S. Dorbolo, Phys. Rev. E 86, 051303 (2012).

[10] https://imagej.nih.gov/ij/

[11] https://www.python.org/ 